\title{
LA ENSEÑANZA DE POLÍTICAS PÚBLICAS POR MEDIO DE UN JUEGO DE ROL. ANÁLISIS DE EXPERIENCIAS EN LA FACULTAD DE CIENCIAS SOCIALES DE LA UNIVERSIDAD DE LA REPÚBLICA (URUGUAY) 2010-2011
}

\author{
Nicolás Bentancur (nicobent@cpolit.edu.uy) \\ Instituto de Ciencia Política \\ Universidad de la República, Uruguay
}

El artículo presenta una experiencia de enseñanza del proceso de elaboración de las políticas públicas en el nivel universitario, empleando una estrategia de juego de rol como recurso didáctico. En la representación del juego de rol, grupos de estudiantes actuaron como actores interesados en una política específica y los docentes asumieron la función de gobernantes. Se sostiene que, de la experiencia han surgido indicios positivos sobre su potencial como herramienta pedagógica, de modo de ambientar una mejor comprensión de la complejidad inherente a un policy making que pretenda generar políticas públicas legitimadas y eficaces.

Palabras clave: enseñanza; juego de rol; politicas públicas. 
TEACHING PUBLIC POLICY THROUGH ROLE-PLAYING. AN ANALYSIS OF THE EXPERIENCE OF THE FACULTY OF SOCIAL SCIENCE OF THE UNIVERSIDAD DE LA REPÚBLICA (URUGUAY): 2010-2011

\begin{abstract}
The article presents a case of teaching public policy creation at the university level, utilizing a role-playing as a didactic tool. Through role-playing, student groups simulate actors interested in a specific public policy and teachers take on the role of government officials. From this experience positive signs may be identified regarding the approach's usefulness as a teaching tool, with a view towards generating greater understanding of the complexity involved in the process of creating legitimate and efficient public policy.
\end{abstract}

Keywords: teaching: role-playing: public policy. 


\section{LOS OBJETIVOS DE UNA MODALIDAD PARTICIPATIVA DE ENSEÑANZA-APRENDIZAJE DE LAS POLÍTICAS PÚBLICAS}

Frecuentemente, la enseñanza de los procesos de políticas públicas en el nivel universitario, especialmente -pero no exclusivamente- en sus primeros ciclos, se enfrenta a la dificultad de trasmitir a los estudiantes la complejidad de las racionalidades, valores e intereses involucrados en el proceso de toma de decisiones. La tradicional y capital distinción entre una modalidad de elaboración racionalista de las políticas, en las que se ponderan todas las alternativas disponibles de acuerdo a una estructura de preferencias bien definida de cada actor y a una estimación precisa de la medida en que cada alternativa impactará en los resultados de la política, por un lado; y una perspectiva incrementalista, según la cual las decisiones suelen reducirse a la ponderación de opciones de política que sólo difieren marginalmente de otras ya en curso, y a su convalidación por medio del acuerdo entre cierto grupo de participantes dotados de recursos de poder suficientes, por lo general es recibida por los alumnos alejados de la práctica de las políticas como una disquisición predominantemente intelectual. En cambio, esos mismos alumnos acostumbran percibir al proceso de decisión bien como una operación básicamente técnica de elección de una política que contribuirá a la obtención del bienestar general, o en el otro extremo, como una simple y directa traducción de las orientaciones ideológicas y programáticas de los gobiernos de turno.

Para superar esas visiones simplistas hemos realizado la experiencia de colocar a grupos de dichos estudiantes en el rol de varios de los actores protagónicos de las políticas públicas: integrantes del partido de gobierno o de oposición, de la alta burocracia, de cámaras empresariales, de sindicatos de trabajadores o de consultores contratados. ¿Cómo razonarían y actuarían ellos mismos, entonces, en un armado participativo y negociado de una política que les afecta? ¿Priorizarían el interés público o sus intereses particulares? ¿Apelarían a argumentos de naturaleza técnica o a los valores involucrados para defender sus posiciones y pretender persuadir a los otros grupos de su legitimidad y conveniencia? ¿Se involucrarían en negociaciones definidas por la afinidad ideológica de sus contrapartes, o actuarían pragmáticamente? Se ha apostado, entonces, a que mediante un proceso intelectual de naturaleza inductiva se comprenda mejor la compleja racionalidad 
de la elaboración de las políticas públicas, a partir de las propias vivencias en un ejercicio de simulación.

Dicho esto, entendemos que el ensayo no es relevante solamente como recurso didáctico para favorecer el aprendizaje de grupos específicos de estudiantes, sino que su finalidad se extiende más allá del salón de clase y se vincula con valores cardinales de la organización social. Las políticas públicas en las sociedades modernas son, en gran medida, la expresión más cabal del sistema democrático y de la institución estatal en movimiento. Por ende, su correcta inteligencia coadyuva al ejercicio más pleno de los derechos de ciudadanía, propiciando una participación y fiscalización más calificada y responsable de su itinerario de diseño, implementación y evaluación. A su vez, niveles superiores de comprensión de las múltiples lógicas y racionalidades que se cruzan en su construcción son un requisito necesario para intentar ensanchar los espacios de racionalidad y de negociación creativa que puedan generarse en su proceso, con las consiguientes ganancias colectivas que estas prácticas pueden aparejar. Aunque resulte paradójico, una adecuada intelección de las variables políticas que naturalmente conducen a la impronta gradualista de las políticas públicas y a una cerrada "política de partidarios" donde priman los ajustes mutuos y las concesiones recíprocas, es un pre-requisito indispensable para ensayar alternativas más orientadas a transformaciones sustantivas y a ganancias sociales más extendidas.

En consecuencia con estas intenciones, este artículo analiza la puesta en práctica y desarrollo de esa experiencia de enseńanza-aprendizaje, tras presentar sumariamente algunas bases didácticas, metodológicas y teóricas-conceptuales que la fundamentan.

Más específicamente, se presenta una experiencia de enseñanza del proceso de elaboración de las políticas públicas en el nivel universitario, empleando una estrategia de juego de rol como recurso didáctico. En la representación del juego de rol, grupos de estudiantes actuaron como distintos actores interesados en una política específica, y los docentes asumieron la función de gobernantes. La simulación tuvo como objetivo favorecer la reflexión y el entendimiento de los estudiantes sobre las variables políticas que inciden en ese proceso, destacando especialmente la existencia de un conjunto de actores políticos y sociales con intereses diferenciados y de un gobierno que -además de actuar como negociador y árbitro del 
proceso- cuenta también con sus propias preferencias. De la experiencia que se describe han emergido indicios positivos sobre su potencial como herramienta pedagógica, de modo de ambientar una mejor comprensión de la complejidad inherente a un policy making que pretenda propiciar políticas públicas legitimadas y eficaces.

\section{EL JUEGO DE ROL COMO ESTRATEGIA DE ENSEÑANZA- APRENDIZAJE EN LAS CIENCIAS SOCIALES}

El recurso didáctico del juego de rol forma parte de la estrategia más amplia de empleo de juegos y simulaciones para la formación en distintas áreas de las ciencias sociales. Las diversas modalidades de simulación tienen por propósito facilitar una comprensión más profunda de los estudiantes de los conceptos, procesos y racionalidades de un campo determinado, haciéndolos trabajar y reflexionar sobre la representación de un entorno real. Esta apuesta ostenta una trayectoria considerable, especialmente en el mundo anglosajón y en disciplinas tales como la economía y las relaciones internacionales. Aunque comparativamente en menor número, también existen aplicaciones en el campo de las políticas públicas (Assal y Blake, 2006; Baranowski, 2006; Endersby y Webber, 1995, Lay y Smarick, 2006; O'Reilly, 2008).

El juego de rol supone, por su parte, una modalidad particular de la simulación. En él, los estudiantes se transforman en actores ("jugadores") que deben actuar de la manera que ellos juzgan apropiada según el rol que deben desempeñar. Si bien deben ajustarse a reglas, como en toda simulación, en este caso los estudiantes gozan de un albedrío más amplio para actuar y tomar decisiones, de acuerdo a su propia interpretación y estrategia a aplicar al escenario virtual predefinido. Por otra parte, si bien es un juego, no necesariamente es uno de suma cero; esto es, puede ambientar tanto modalidades de actuación competitivas como colaborativas. Según Gil (2010), las características del juego de rol son las siguientes:

- Reducen una situación real a la escala del aula, haciendo su complejidad abarcable para los alumnos.

- Plantean una acción que implica y motiva a los participantes. 
- Dan la posibilidad de trabajar sobre sistemas sociales complejos.

- Permiten trabajar múltiples aspectos actitudinales: tolerancia, empatía, respeto, trabajo en grupo, espíritu crítico, correcta expresión, capacidad de síntesis.

- Desarrollan la capacidad de expresión y comprensión.

- Introducen una ruptura con el ritmo habitual de las clases.

De acuerdo a la misma autora (Gil, 2010), el juego de rol apunta a los siguientes objetivos:

- Ayudar a examinar problemas reales a nivel teórico, emocional y físico.

- Probar y analizar situaciones, teorías y tácticas.

- Comprender a las personas y el papel que desempeñan.

- Entender los pensamientos y sentimientos de las personas "oponentes".

- Anticiparse a nuevas situaciones.

- Sacar fuera temores, ansiedades y otros sentimientos que las personas suelen tener ante una acción.

- Conseguir más información.

- Desarrollar la cohesión de grupo.

- Aprender nuevas destrezas ante ciertas situaciones y experimentar su utilización.

- Adquirir confianza y competencia individual y grupal.

Ahora bien, ¿qué factores abonarían la conveniencia de empleo de esta modalidad didáctica? Luego de pasar revista a los aportes de varios académicos que abordaron el tópico, Sutcliffe (2011) identifica los siguientes:

- Impulsar a los estudiantes a reflexionar sobre sus conocimientos y a extraer conjuntamente conclusiones sobre los temas planteados en el curso.

- Favorecer una mejor apreciación de las características de los distintos roles y de sus responsabilidades, desarrollando la empatía de los estudiantes con diferentes posturas y sentimientos más allá de sus propias asunciones y expectativas. 
- Contribuir a una mejor dinámica grupal, favoreciendo la interactividad.

- Otorgar "vida" a conceptos teóricos abstractos, colocándolos en situaciones que les resultan familiares a los intérpretes.

- Contribuir al desarrollo de destrezas clave, en términos de habilidades comunicativas y sociales.

Existen numerosas experiencias que avalan el rédito de estos mecanismos en el aprendizaje de otras ciencias sociales (ver Sutcliffe, 2011), y se dispone incluso de algunas investigaciones realizadas con el empleo de métodos estadísticos, que dan cuenta de la buena valoración que hacen estudiantes de pregrado universitario de esta herramienta de enseńanza-aprendizaje (Bolzan, 2003; Ferreira Borges, 2011). Ello ha alentado nuestro ensayo en la sub-disciplina de las políticas públicas, en cursos de licenciatura del Ciclo Inicial de la Facultad de Ciencias Sociales de Uruguay.

\section{ALGUNAS PREMISAS TEÓRICO-CONCEPTUALES PARA EL ANÁLISIS DE LAS POLÍTICAS PÚBLICAS}

Naturalmente, la adscripción de este juego de rol a un curso académico requirió la explicitación de ciertos desarrollos de la teoría de las políticas públicas en algunas sesiones de clases previas, y en otras concomitantes a la experiencia. En la medida en que se apuntó a la evaluación crítica de esos desarrollos, se los planteó como conceptos sujetos a verificación y "falseamiento" en la propia dinámica a desarrollar. Entre esos conceptos se hallaban:

a) La concepción pluralista de la política pública como un "juego de poder", en el que participan múltiples actores con valores, intereses, estrategias y recursos diferentes (Lindblom, 1991), si bien corregida asignando un mayor protagonismo al gobierno que el que le atribuye esta corriente teórica, según sugieren otras indagaciones (Grindle y Thomas, 1991);

b) La teoría de la elección pública, que concibe a los decisores de políticas como maximizadores de utilidades y orientados por la prosecución de intereses 
particulares, sean éstos funcionarios (Niskanen, 1980) o partidos políticos (Downs, 1992).

c) La conceptualización de que los colectivos participantes en la formulación de las políticas desarrollan determinadas estrategias (Lindblom, 1991) en las que movilizan recursos de poder específicos a su disposición (Subirats et al., 2008).

d) La existencia de actores con "poder de veto" sobre alternativas decisorias que no le satisfacen en ciertas políticas sectoriales, y por ende, cuya adhesión es requerida para alterar el statuquo (Tsebelis, 2000).

e) El postulado de que los partidos políticos, además de los intereses de conservación y ampliación de sus espacios de poder institucionalizado, se movilizan también de acuerdo a orientaciones ideológicas y programáticas que inciden en el direccionamiento de las políticas públicas que propugnan (Boix, 2002).

\section{EL DISEÑO DEL JUEGO DE ROL}

Las reglas detalladas del juego se incorporan en el Anexo de este informe. En líneas generales, los elementos más destacados de la simulación fueron los siguientes:

Se seleccionó una política sectorial (un plan de vivienda) y a partidos políticos que en su definición guardan una relación aproximada con sus referentes reales del país en que se realizó el ejercicio (Uruguay) en los años 2010 y 2011, a efectos de otorgarle visos de realidad. Básicamente, el caso es el siguiente: la definición de una política de vivienda destinada a los sectores de menores ingresos de la población, en la que distintos actores políticos y sociales deberán establecer sus características en algunas dimensiones fundamentales.

Estas dimensiones o ejes centrales en cuestión son los siguientes:

a) su modalidad de construcción, y el protagonismo relativo de distintos actores: ¿se construirán bajo la dirección y con personal del aparato burocrático estatal, se confiarán a empresas privadas o contarán con un fuerte componente de voluntariado social? 
b) el tiempo de construcción: ¿se apostará a soluciones habitacionales de rápida concreción, o a alternativas superiores que demorarían la respuesta a la necesidad social?

c) la estrategia de financiamiento, para obtener los recursos necesarios para solventar el programa: ¿̇e aumentará la carga impositiva, se recortarán y transferirán otros rubros del gasto público o se acudirá a préstamos externos?

d) la definición del tipo de vivienda a construir: ¿cómo se balancearán los valores contrapuestos de la calidad de las viviendas, por un lado, y de la cantidad a construir, dado un presupuesto acotado?

En la simulación, además del gobierno, se identificaron seis actores: el partido de gobierno, una coalición de partidos de oposición, la alta burocracia de un Ministerio de Vivienda, una gremial de empresas de la construcción, el sindicato de trabajadores del sector, e intelectuales que asesoraban en la toma de decisiones. Se dividió a los estudiantes en seis grupos que desempeñaron el rol de cada uno de esos actores (asignados por su propia elección, y en su defecto, por sorteo). Para cada uno de ellos se explicitó un perfil de orientación ideológico-programática y de intereses particulares (necesariamente caricaturizados con fines didácticos), a los que se asoció una determinada estructura racional de preferencias en cuatro "ejes de política" a resolver (la modalidad de construcción de las viviendas, el plazo de ejecución, las fuentes de financiamiento, el tipo de vivienda a construir).

Se asignó a cada actor-grupo un determinado número de "votos" o "recursos" que representaban su poder de influencia en la decisión de la política, que podían distribuir de la manera que prefirieran en la incidencia (votación) de los cuatro ejes de políticas. Además, se concedió un solo derecho de "veto" a cada actor. El gobierno como tal (representado por el equipo docente) no votaba, pero manejaba los tiempos de la negociación, disponía cuartos intermedios, fundamentaba posiciones, promovía acuerdos y disponía de dos recursos de veto, que podía usar además para levantar el veto que pudieran interponer otros actores.

La mecánica del juego contemplaba varias fases sucesivas, aplicadas para cada uno de esos cuatro ejes: articulación de intereses (definición de estrategias intra-grupos), argumentación pública a favor de su opción de política preferida, agregación de intereses (negociación intergrupal), votación plenaria, recuento de 
recursos/votos, y (eventualmente) aplicación de vetos a la decisión que recogió la mayoría de los votos. Cada opción vetada era descartada, y se volvía a desarrollar todo el proceso, culminando en una nueva votación sobre las opciones restantes.

Al final de la resolución de cada uno de los ejes de políticas, se imputaba a cada actor determinada cantidad de "puntos", acorde con lo favorable que le resultó la decisión adoptada, según su estructura de preferencias predeterminadas.

El juego culminaba cuando se decidiera sobre los cuatro ejes de políticas, y en ese caso se realizaba una ordenación final de los actores según los puntos obtenidos; o bien, cuando se truncara el proceso de formulación de la política a causa de su obstrucción por sendos vetos interpuestos contra todas las opciones disponibles en uno cualquiera de los ejes a decidir. En este caso, la política quedaría sin efecto y todos los actores perderían.

\section{EL DESEMPEÑO EFECTIVO DE LOS GRUPOS ESTUDIANTILES EN EL JUEGO DE ROL}

La simulación se realizó en dos años sucesivos (2010 y 2011) con resultados diferentes: en el primer caso, grupos de estudiantes que no lograron sus objetivos en la definición de los primeros ejes lograron obstruir completamente el desarrollo de la política, constituyendo por tanto un juego colectivo de suma negativa. En el segundo, la política llegó a término, luego de sobreponerse a varios intentos de obstrucción.

En ambos casos, los estudiantes encarnaron con toda fidelidad y entusiasmo la identidad de los actores que representaban, procurando en cada fundamentación, negociación y votación maximizar sus recompensas, con independencia de cualquier reflexión sobre intereses más generales no explicitados (pero evidentes en una política de vivienda focalizada), y en ocasiones en desmedro del perfil ideológico del mismo actor (por ejemplo, el "sindicato de trabajadores de la construcción” intentó vetar toda esta política, de indudable interés social, cuando sus preferencias no se vieron contempladas). Por lo común, las negociaciones entre grupos tampoco tuvieron como referencia su afinidad ideológica, sino simplemente intereses comunes en las votaciones. En ninguna ocasión se laudó un eje 
por argumentos contrastados por evidencia empírica acerca de la conveniencia de una u otra solución, sino que la fundamentación de las ventajas o desventajas de ellas se defendió pretendiendo legitimarlas en base a presuntos intereses nacionales, que siempre coincidían con el interés de cada actor. Un elemento clave en la simulación es que en ninguna parte de las reglas de juego se indicó que existirían "ganadores", y menos aún que estos serían aquellos que maximizaran el interés particular: la asunción de esta supuesta pauta no explicitada fue espontánea, y orientó toda la estrategia desarrollada por los estudiantes ${ }^{I}$.

Ningún grupo apostó a estrategias que privilegiaran supuestos intereses generales, con independencia de sus roles, procurando otra forma de satisfacción que no fuera la del interés particular.

\section{TRAMITACIÓN POSTERIOR AL JUEGO DE ROL Y RESULTADOS DE APRENDIZAJE}

Una vez finalizado el juego, se invitó a reflexionar grupalmente acerca del desarrollo de la experiencia, y especialmente, de los móviles que impulsaron a la adopción de las estrategias referidas. A posteriori, se les indicó la profundización del análisis poniendo en relación los aportes teóricos volcados en otras clases del curso y la simulación, por medio de una monografía externa de carácter grupal, que tiene como pautas la movilización y contrastación de las premisas teóricoconceptuales manejadas, y de preguntas disparadoras de la reflexión, como las siguientes:

- ¿Por qué consideran que se llegaron a tomar las decisiones adoptadas?

- ¿Entienden que los distintos grupos actuaron racionalmente?

- ¿Privilegiaron con su accionar el interés general o intereses particulares?

- ¿Se negoció eficazmente para lograr un equilibrio de ambos términos?

1 Igualmente se dejó claro desde el principio que la puntuación que se recogiera no tendría ninguna incidencia en la calificación del curso. 
- ¿Por qué algunos actores obtuvieron más beneficios ("puntos") que otros? ¿Los que obtuvieron menos beneficios, cómo deberían haber corregido sus prácticas para lograr un resultado positivo?

- ¿Qué conclusiones han extraído de la experiencia en cuanto a las posibilidades y condiciones deseables para obtener políticas públicas "legitimadas" y de "equidad pertinente" (Repetto, 2004)?

- ¿La práctica sugiere que convendría sacrificar parcialmente una finalidad en beneficio de otra (por ejemplo, políticas menos participativas pero pretendidamente más eficaces)?

Una ponderación confiable del aprendizaje efectivo derivado de la experiencia deberá aguardar a su repetición en venideras ediciones del curso, con el instrumental de medición apropiado. No obstante, tanto de la propia evaluación que los estudiantes realizan de la experiencia, como del análisis compartido luego de su realización y de las observaciones y reflexiones incluidas en los trabajos monográficos, se evidencia preliminarmente una comprensión de las elaboraciones teóricas y de la sustancia y complejidad del proceso de toma de decisiones en las políticas públicas mucho más cercana a la realidad que el que detentaban los mismos participantes al comienzo del curso, y de mayor rendimiento que el obtenido en otras versiones de cursos similares que emplearon estrategias más clásicas de enseñanza. Particularmente, los estudiantes comprendieron más cabalmente, entre otros tópicos:

- las razones del pragmatismo que suele orientar el proceso de elaboración de las políticas públicas en sociedades democráticas y plurales, que abonan en términos generales las explicaciones incrementalistas de estas hechuras;

- la complejidad de identificar un "interés general” más allá de las preferencias de los diversos grupos;

- la importancia y la necesidad de la negociación entre actores, más allá de la distancia ideológica o programática existente entre ellos;

- la dificultad para tomar decisiones basadas exclusivamente en valores, en un marco de constricciones políticas, financieras y temporales; 
- las limitaciones efectivas del conocimiento para dirimir cuestiones (y en cambio, su permanente utilización con fines partidaristas);

- la tensión existente entre hechuras de políticas más inclusivas y otras supuestamente más "eficaces" para el logro de objetivos que se reputan valiosos.

\section{A MODO DE CONCLUSIÓN}

Resultó bastante evidente que los estudiantes habían logrado una compresión más cercana a la complejidad del policy making y especialmente a su naturaleza fundamentalmente política, y desarrollado una mayor empatía con los actores políticos y sociales más relevantes. A partir de ese punto de comprensión, el cierre del curso por parte del equipo docente hizo énfasis en dos aspectos:

Por un lado, el reconocimiento de dos limitaciones fundamentales de la simulación: i) la carencia de una dimensión temporal más extendida (en la cual la confianza o desconfianza entre los actores jugaría un papel importante, y por tanto condicionaría las interacciones), y ii) la reducción del juego a una sola política, mientras en la realidad los distintos issues se tramitan simultáneamente, complejizando el juego y condicionando las estrategias de los actores.

Por otro, es posible conjugar el relativismo inherente a varios de los asertos que emergieron de la simulación con la necesidad de construir - pese a todo- prácticas políticas que favorezcan juegos de suma positiva y prohíjen, por tanto, mejores políticas públicas. Esto es, la necesidad de desarrollar desde el reconocimiento de la multiplicidad de actores con visiones e intereses divergentes que tradicionalmente han descripto las corrientes pluralistas-incrementalistas, formatos y experiencias que maximicen las estrategias negociadoras (Fisher y Ury, 1985) y el uso del conocimiento (Dror, 1989) para resolver problemas colectivos.

Por tanto, entendemos que esta experiencia de juego de rol -con los aprendizajes y adaptaciones que correspondan- es una alternativa digna de consideración a la hora de plantearse la enseńanza en este campo. 


\section{REFERENCIAS BIBLIOGRÁFICAS}

Asal, Victor y Blake, Elizabeth (2006). "Creating Simulations for Political Science Education". Journal of Political Science Education Vol. 2, № 1, pp. 1-18.

Baranowski, Michael (2006). "Single Session Simulations: The Effectiveness of Short Congressional Simulations in Introductory American Government Classes”. Journal of Political Science Education Vol. 2, No 1, pp. 33-49.

Boix, Carles (2002). Partidos politicos, crecimiento e igualdad. Madrid: Alianza.

Bolzan, R. F. F. A. (2003). “O aprendizado na internet utilizando estratégias de roleplaying game (RPG)”. Tesis de doctorado, Universidade Federal de Santa Catarina, Florianópolis, Brasil.

Downs, Anthony (1992). “Teoría económica de la acción política en una democracia”, en G. Almond et al. Diez Textos Básicos de Ciencia Política. Barcelona: Ariel Ciencia Política.

Dror, Yehezkel (1989). Public Policymaking reexamined. New Brunswick and Oxford: Transaction Publishers.

Endersby, James W. y David J. Webber (1995). “Iron Triangle Simulation: A role-playing game for undergraduates in Congress, interest groups, and public policy classes”. PS: Political Science and Politics, vol. 28, No3, pp. 520-523.

Ferreira Borges, Aurelio et al. (2011). "Estrategias de enseñanza-aprendizaje con el apoyo del juego pedagógico roleplaying game”. Revista Iberoamericana de Educación, Vol. 56, № 3.

Fisher, Roger y Ury, William (1985). Sí, de acuerdo! Cómo negociar sin ceder. Bogotá: Norma.

Gil, Carolina (2010). "El juego de rol aplicado a la educación ambiental”. Disponible al 2.9.2011 en http://www2.fe.ccoo.es/andalucia/docu/p5sd7055.pdf

Grindle, Merilee S. y Thomas, John W. (1991). Public choices and policy change. The political economy of reforms in developing countries. Baltimore: The Johns Hopkins University Press.

Lay, Celeste J., y Kathleen J. Smarick (2006). "Simulating a Senate Office: The Impact on Student Knowledge and Attitudes”. Journal of Political Science Education Vol. 2 No 2, pp. 131-146

Lindblom, Charles (1991). El proceso de elaboración de las políticas públicas. México DC: Porrúa.

Niskanen, William (1980). Cara y cruz de la burocracia. Madrid: Espasa Calpe.

O'Reilly, Patricia (2008). "Finding the balance in public policy simulations and role playing”. Paper presentado al Anual Meeting of the APSA Teaching and Learning Conference, San José, California. 
Repetto, F. (2004). Capacidad estatal. Requisito para el mejoramiento de la política social en América Latina. Documentos de trabajo del INDES.

Subirats, J. et al. (2008). Análisis y gestión de políticas públicas. Barcelona: Ariel.

Sutcliffe, Mark. "Simulations, games and role - play". Disponible al 2.9.2011 en www.economicsnetwork.ac.uk/handbook/printable/games_vs.pdf

Tsebelis, George (2000). "Veto players and institutional analysis”, en Governance, No 13, pp. 441474.

\section{ANEXO}

\section{EL JUEGO DE LAS POLÍTICAS PÚBLICAS: SIMULACIÓN DE ROLES Y DE PROCESO DECISORIO}

\section{A) MECÁNICA DE LA SIMULACIÓN}

I) Objetivo del Juego: Lograr una decisión de política pública lo más satisfactoria posible para el actor político, representado por cada uno de los grupos de estudiantes.

\section{II) Reglas del juego:}

a) Se propone un caso simulado de política pública a resolver, en la que se identifican como actores principales al gobierno nacional y a seis actores más, interactuando en una "mesa de negociación" especialmente convocada a esos efectos. El rol del gobierno será asumido por los docentes, el resto será distribuido aleatoriamente entre grupos de estudiantes.

b) Se identifican cuatro ejes centrales sobre los que debe resolverse, que resultan de importancia variable para cada uno de los actores. En cada uno de esos ejes, se proponen distintas alternativas de decisión, una de las cuales debe ser seleccionada por los participantes al finalizar su tratamiento.

c) De acuerdo a sus concepciones, valores e intereses, cada uno de los actores tiene una estructura de preferencias que se predeterminan; esto es, actúan según una priorización de cada alternativa de decisión dentro de cada eje de política. 
d) Cada actor (grupo) cuenta con cierta dotación de recursos de poder cuantificada y no renovable (que a los efectos de simplificar la simulación no se desagregan en sus distintos tipos). La alternativa elegida (decisión) en cada eje de políticas será aquella que al momento de la votación cuente con el apoyo de la mayor cantidad de unidades de recursos, previo a lo cual pueden establecerse acuerdos entre dos o más actores fuera de la reunión plenaria de la comisión. Cada actor puede invertir todo o parte de sus recursos en una o más votaciones.

e) La dinámica del juego supone las siguientes fases, a reiterar para la decisión en cada uno de esos cuatro ejes de políticas:

- Articulación de intereses. Definición de la estrategia a llevar adelante por cada actor, en reunión cerrada del grupo.

- Argumentación. Defensa pública, en régimen de plenario, de la solución preferida por cada actor con argumentos valorativos y (de ser posible) datos empíricos, procurando ganar aliados y convencer al gobierno.

- Agregación de intereses. Negociación libre y reservada entre actores sobre la posterior votación.

- Votación plenaria. Asignación de recursos de cada actor a cada una de las alternativas de decisión (la votación de cada actor no será revelada públicamente).

- Recuento de recursos/votos. La opción que haya recibido la cantidad mayor será, en primera instancia, la escogida para el eje de políticas considerado.

- Veto: a) luego de la votación de cada eje, cada actor tendrá la posibilidad de vetar, públicamente, la resolución tomada, salvo que la misma haya sido adoptada por unanimidad, o haya recibido previamente el voto del mismo actor. Cada actor sólo podrá interponer un veto a lo largo de todo el juego. b) Por excepción, el gobierno puede vetar hasta dos veces; y a diferencia de los otros actores, puede usar su veto para levantar el veto interpuesto por otro actor. Además, el gobierno puede decidir interrupciones y postergaciones de la discusión y de las votaciones. 
c) La interposición del veto supone una nueva ronda de argumentación, negociación y votación, en la que se descarta la opción vetada. Los recursos aplicados en la votación anterior se devuelven a los grupos.

d) Si en alguno de los ejes de políticas se eliminaran todas las opciones, el proceso de elaboración de la política se frustra, culmina el juego y todos los actores pierden los puntos obtenidos.

III) Resolución del juego: Al final del juego, los actores se ordenarán de acuerdo a los puntos obtenidos.

IV) Evaluación de la simulación para el curso: en la última sesión, los docentes propondrán a los diferentes grupos un cuestionario para el análisis de la experiencia, que deberán contestar haciendo referencia a los procesos desarrollados y empleando la bibliografía del curso. Este trabajo representará la tercera parte de la calificación del segundo parcial.

\section{B) EL CASO DE POLÍTICA A DESARROLLAR}

Por tratarse de un ejercicio con finalidades formativas, se simplifican y esquematizan algunas referencias de la política nacional. Particularmente, no se pretende que la caracterización y las preferencias de los actores se ajusten estrictamente a la realidad.

\section{Objetivo de la política pública y financiamiento}

Formular una política de vivienda que tiene por objetivo general la construcción de soluciones habitacionales para familias de escasos recursos, para lo que puede destinarse hasta $\$ 1.000$ por año.

\section{Escenario}

Para la formulación de dicha política, el partido de gobierno convoca a una mesa de negociación de los principales actores involucrados, de donde saldrá el proyecto de ley a enviar al parlamento.

\section{Principales ejes de la política pública y alternativas de decisión}

La política pública de vivienda que el gobierno pretende formular, tiene 4 ejes principales, con sus respectivas alternativas de decisión, a saber: 
1) Modalidad de construcción de las viviendas

a) Empleando funcionarios públicos de planta o a contratar por los ministerios.

b) Tercerización, mediante empresas privadas.

c) Empleando funcionarios públicos en colaboración con personal voluntario.

2) Variable temporal - Plazo para finalizar la construcción de las viviendas
a) 1 año
b) 2 años
c) 3 años
d) 4 años

3) Modalidad de financiamiento

a) Combinación de recortes de gastos del Estado (de otros ítems presupuestarios) y endeudamiento externo (préstamo de IFI).

b) Combinación entre aumento de impuestos y endeudamiento externo (préstamo de IFI).

c) Únicamente mediante recorte de otros rubros del gasto público.

d) Únicamente a través de endeudamiento externo.

4) Tipo de vivienda

a) Viviendas pre-fabricadas de dos dormitorios (costo total \$ 1000). Vida útil estimada: 25 años.

b) Viviendas del tipo "núcleo básico evolutivo" (costo total \$2000). Consiste en una construcción básica de material, de dos dormitorios, ampliable posteriormente.

c) Viviendas estándar de dos dormitorios (costo total \$ 4000)

\section{Actores y recursos de poder:}

1. Partido de gobierno. Recursos de poder: 100.

2. Principal partido de oposición. Recursos de poder: 60 . 
3. Miembros de la alta burocracia del Ministerio directamente involucrado (Vivienda). Recursos de poder: 35 .

4. Analistas simbólicos o técnicos (son contratados y financiados por un organismo de financiamiento internacional). Recursos de poder: 35.

5. Cámara que reúne a los empresarios de la construcción. Recursos de poder: 35.

6. Sindicato de los trabajadores de la construcción. Recursos de poder: 35.

\section{Caracterización de los actores:}

1. Partido de gobierno: Orientación ideológica: centroizquierda, predominantemente estatista. La construcción de viviendas de este tipo es una de sus principales promesas electorales y líneas programáticas. Concibe esta política como una política social, habiéndose comprometido a ofrecer rápidamente solución a los problemas habitacionales de las familias con escasos recursos. En cambio, los posibles efectos positivos secundarios en la economía de la construcción de estas viviendas (empleo, demanda agregada) no constituyeron un elemento central al definir esta política en la plataforma de gobierno.

2. Principal Partido de Oposición: Orientación ideológica: centroderecha, predominantemente liberal. Políticamente, su objetivo central radica en recuperar el gobierno en las próximas elecciones, dentro de poco más de 4 años. Sus principales orientaciones en términos de política económica están centradas en la reducción del gasto público y en no aumentar la carga impositiva. Tiene una visión favorable a un rol activo de los actores privados en la economía, mientras adjudica un rol secundario al Estado como productor de bienes y servicios. En relación a la política de vivienda, comparte el interés gubernamental en la erradicación de asentamientos.

3. Alta Burocracia (Ministerio de Vivienda): El personal de carrera superior de estos ministerios busca en primer lugar preservar su propia capacidad de dirección y decisión, basada en su sitial en el organigrama estatal y en sus conocimientos específicos. Asimismo, pretende desarrollar un rol protagónico en las políticas sectoriales que le atañen, como estrategia para incrementar sus competencias, el monto de la ejecución presupuestal a su cargo y su reconocimiento y legitimidad. 
Dado su perfil técnico y su apego a las normas de procedimiento, prefiere una modalidad de formulación de políticas públicas que contemple la realización de un adecuado diagnóstico previo, aunque ello postergue su ejecución.

4. Analistas simbólicos/técnicos: Este grupo de especialistas es contratado a término por una institución financiera internacional (IFI), como colaboración con el país para el diseńo de la política. Su permanencia como personal contratado por dicha institución, para tareas de monitoreo y evaluación, dependerá del tipo de decisión que se adopte. Debido a la experticia profesional de dicho grupo, en su mayor parte integrado por economistas, privilegia el análisis costo-beneficio para ponderar las distintas alternativas de políticas.

5. Cámara que reúne a los empresarios de la construcción: Conviene a sus intereses económicos tener la mayor participación posible (en duración y/o recursos financieros a obtener) en la ejecución del plan de vivienda.

6. Sindicato de los trabajadores de la construcción: Su objetivo principal en esta línea es la obtención de empleo por el mayor plazo posible para sus afiliados. Por su extracción social y orientación ideológica, están además comprometidos con el éxito de la política a decidir.

\section{Importancia relativa de los ejes para los actores}

De acuerdo a la caracterización de cada actor antes definida, la importancia que le dan a cada eje de política es variable. En el cuadro 1 se pondera esa importancia relativa en una escala de 1 a 10 puntos. Cada puntaje representa también el valor máximo que puede obtener cada actor en un eje de políticas. Para equilibrar a los actores en el juego, se supone que el nivel de interés de cada uno de ellos por el conjunto de los ejes de política es similar (se fija en 25 puntos). 


\section{Cuadro 1}

\begin{tabular}{l|c|c|c|c|c}
\multicolumn{1}{c|}{ Actores } & $\begin{array}{c}\text { Eje 1: } \\
\text { Modalidad } \\
\text { de construcción }\end{array}$ & $\begin{array}{c}\text { Eje 2: } \\
\text { Plazo } \\
\text { de ejecución }\end{array}$ & $\begin{array}{c}\text { Eje 3: } \\
\text { Modalidad } \\
\text { de financiam. }\end{array}$ & $\begin{array}{c}\text { Eje 4: } \\
\text { Tipo } \\
\text { de vivienda }\end{array}$ & Totales \\
\hline Partido de Gobierno & 3 & 10 & 5 & 7 & 25 \\
\hline Partido de Oposición & 7 & 4 & 10 & 4 & 25 \\
\hline Alta Burocracia & 10 & 7 & 2 & 6 & 25 \\
\hline $\begin{array}{l}\text { Analistas simbólicos/ } \\
\text { técnicos IFI. }\end{array}$ & 7 & 8 & 6 & 4 & 25 \\
\hline $\begin{array}{l}\text { Empresarios } \\
\text { de la construcción }\end{array}$ & 10 & 7 & 4 & 4 & 25 \\
\hline $\begin{array}{l}\text { Sindicato } \\
\text { de la construcción }\end{array}$ & 10 & 8 & 3 & 4 & 25 \\
\hline \begin{tabular}{l} 
Valores totales \\
\hline
\end{tabular} & 47 & 44 & 30 & 29 & 150 \\
\hline
\end{tabular}

\section{Estructura de preferencias de los actores y retribuciones de las decisiones}

En consonancia con la caracterización de cada actor, en el cuadro 2 se consignan los puntos que obtendrían si se seleccionaran las distintas alternativas de cada eje.

Cuadro 2

\begin{tabular}{|c|c|c|c|c|c|c|c|c|c|c|c|c|c|c|}
\hline \multirow[t]{2}{*}{ Actores } & \multicolumn{3}{|c|}{$\begin{array}{c}\text { Eje 1: } \\
\text { Modalidad } \\
\text { de construc- } \\
\text { ción } \\
\end{array}$} & \multicolumn{4}{|c|}{$\begin{array}{c}\text { Eje 2: } \\
\text { Plazo } \\
\text { de ejecución }\end{array}$} & \multicolumn{4}{|c|}{$\begin{array}{c}\text { Eje 3: } \\
\text { Modalidad } \\
\text { de financiamiento }\end{array}$} & \multicolumn{3}{|c|}{$\begin{array}{c}\text { Eje 4: } \\
\text { Tipo de } \\
\text { vivienda }\end{array}$} \\
\hline & a & $\mathrm{b}$ & c & a & B & c & d & a & $\mathrm{b}$ & c & d & A & $\mathrm{b}$ & c \\
\hline Partido de Gobierno & 2 & 1 & 3 & 10 & 7 & 4 & 2 & 5 & 2 & 1 & 4 & 4 & 6 & 7 \\
\hline Coalición de Oposición & 3 & 7 & 2 & 1 & 2 & 3 & 4 & 7 & 2 & 10 & 4 & 2 & 2 & 4 \\
\hline Alta Burocracia & 10 & 1 & 7 & 2 & 3 & 5 & 7 & 1 & 2 & 1 & 2 & 2 & 4 & 6 \\
\hline Analistas simbólicos/ técnicos & 2 & 7 & 5 & 2 & 4 & 6 & 8 & 6 & 2 & 4 & 2 & 2 & 4 & 2 \\
\hline Empresarios de la construcción & 4 & 10 & 4 & 2 & 4 & 6 & 7 & 4 & 1 & 2 & 3 & 1 & 2 & 4 \\
\hline Sindicato de la construcción & 1 & 10 & 2 & 2 & 4 & 6 & 8 & 2 & 3 & 1 & 2 & 1 & 2 & 4 \\
\hline
\end{tabular}

Recibido: 25 agosto 2011 / Aprobado: 15 noviembre 2011 